\title{
A Model for Asymmetric Hysteresis in Piezoceramic Materials
}

\author{
Ralph C. Smith \\ Center for Research in Scientific Computation \\ Department of Mathematics \\ North Carolina State University \\ Raleigh, NC 27695-8205 \\ rsmith@eos.ncsu.edu \\ Zoubeida Ounaies \\ ICASE, M/S 132C \\ NASA Langley Research Center \\ Hampton, VA 23681-2199 \\ z.ounaies@larc.nasa.gov
}

\begin{abstract}
This paper focuses on the characterization of hysteresis exhibited by piezoelectric materials at moderate to high field levels. For soft materials in which dipoles are easily reconfigured, the hysteresis loop is observed to be rotationally symmetric about the zero field, zero polarization point and symmetric models can be employed. In harder materials, however, the loops are no longer rotationally symmetric which necessitates the development of commensurate characterization techniques. The model considered here is based upon the quantification of reversible and irreversible changes in polarization due to bending and translation of domain walls pinned at inclusions inherent to the materials. The performance of the model is illustrated through comparison with PZT4 data.
\end{abstract}




\section{Introduction}

An inherent property of piezoelectric materials is the presence of hysteresis and constitutive nonlinearities at moderate to high drive levels. In certain applications, the degree of hysteresis can be minimized by restricting input field or stress levels, or employing certain feedback mechanisms [7]. However, to attain the full capabilities of the materials in high performance applications, it is necessary to quantify the hysteresis and nonlinearities in a manner which permits both material characterization and efficient control design. The latter criterion is facilitated by the development of models which can either be exactly or approximately inverted to permit the construction of inverse compensators for linear control design.

A common form of hysteresis in piezoelectric materials is the sigmoid relation between the input field $E$ and polarization $P$ illustrated in Figure 1. For soft materials in which dipoles are easily reversed, the curves are observed to be approximately rotationally symmetric about the point $E=0$, $P=0$. For harder materials which are initially poled, the relation is often asymmetric due to the fact that the preferred orientation induced during poling is less easily modified by AC fields at normal operating temperatures. Such a response is illustrated in Figure 1a with data from a poled PZT4 wafer. For general applications, it is advantageous to have a model which accommodates both the symmetric unpoled response and asymmetric poled response through the characterization of physical mechanisms which produce the hysteresis. This is accomplished here through the quantification of energy losses which occur when domains reorient and domain walls move in response to an applied

field. Details regarding the associated reversible and irreversible domain wall mechanisms can be found in $[1,6]$.

Various techniques have been employed for modeling hysteresis in piezoelectric materials including macroscopic phenomenological approaches [3, 4, 15], microscopic energy-based models at the lattice or grain level [8], or semi-macroscopic theories which employ energy relations in combination with macroscopic averages to yield models having effective parameters which characterize the bulk behavior of the material [2]. The present model fits in this latter category.

The initial theory underlying this model was developed in $[10,11]$ for general ferroelectric materials where it was illustrated for PMN at low temperatures. This theory quantifies through electrostatic energy relations the reversible and irreversible effects of domain wall bending and translation. Macroscopic averages then provide a model having five parameters which quantifies the bulk attributes of the material. The extension of this model to piezoelectric compounds and validation for PZT5A was provided in [12]. The initial models and validation results accommodate only symmetric hysteresis loops.

The extension of the model to accommodate asymmetric hysteresis loops of the type depicted in Figure 1 is outlined in the next section and then illustrated through a comparison with data from both poled and depoled PZT4 wafers. We note that the basis of the technique on physical mechanisms yields a model in which the effects of poling are accommodated by biases in the field and polarization with the original five hysteresis parameters remaining unchanged. This provides the model with significant flexibility for both material characterization and control design since changes due to poling (or depoling) are incorporated through the physics rather than changes in the model or hysteresis parameters.

\section{Model Development}

The hysteresis between the electric field $E$ and polarization $P$ is modeled in two steps. In the first, the ideal anhysteretic polarization, which would result in the absence of domain wall pinning, is quantified 
by employing Boltzmann statistics to determine the probability of dipoles occupying specified energy states. Hysteresis is then incorporated by computing deviations from the anhysteretic state due the restriction of domain wall movement by pinning sites in the material. This approach is motivated by analogous theory for ferromagnetic materials [5]. An analogous model for symmetric hysteresis loops in ferroelectric materials is summarized in $[10,11]$ with initial validation for piezoelectric materials provided in $[12,13]$.

To characterize the anhysteretic polarization which would result in the absence of pinning sites, it is necessary to consider the effective field acting on dipoles in the material. In the original symmetric models, the effective field was taken to be

$$
E_{e}=E+\alpha P
$$

where the component $\alpha P$ quantifies the field contributions due to interdomain coupling and certain stress effects. As detailed in $[10,11]$, the parameter $\alpha$ can be formulated in terms of a scaling electric field $\widehat{E}$ and the saturation polarization $P_{s}$, but is typically estimated in applications through a least squares fit to data. The asymmetries due to poling are incorporated by noting that they produce a bias field $E_{0}$ and bias polarization $P_{0}$ which causes the positive and negative coercive fields and remanence points to differ. The resulting effective field is then modeled by

$$
E_{e}=\left(E-E_{0}\right)+\alpha\left(P-P_{0}\right) .
$$

As detailed in $[10,11]$, the balancing of electrostatic and thermal energy using Boltzmann principles yields a family of models which differ according to assumptions concerning possible domain orientations. In the presence of a scaling polarization $P_{1}$, the assumption that cells can be oriented in any direction yields the Langevin model

$$
P_{a n}=P_{1}+P_{s}\left[\operatorname{coth}\left(\frac{E_{e}}{a}\right)-\frac{a}{E_{e}}\right]
$$

while the assumption that dipoles can orient only in the direction of the field, or opposite to it, yields the Ising spin model

$$
P_{a n}=P_{1}+P_{s} \tanh \left(\frac{E_{e}}{a}\right) .
$$

In both cases, $P_{s}$ denotes the saturation polarization while $a$ is a parameter which is estimated through a least squares fit to data. Details regarding the behavior of these two models can be found in $[10,11]$.

Hysteresis is incorporated by computing the irreversible polarization $P_{i r r}$ and reversible polarization $P_{\text {rev }}$ resulting from the translation and bending of domain walls pinned at inclusions in the material. The quantification of energy required to break pinning sites yields the differential equation

$$
\frac{d P_{i r r}}{d E}=\widetilde{\delta} \frac{P_{a n}-P_{i r r}}{k \delta-\alpha\left(P_{a n}-P_{i r r}\right)}
$$

specifying the irreversible polarization. The parameter $\delta=\operatorname{sign}(d E)$ ensures that the energy required to break pinning sites always opposes changes in polarization while

$$
\widetilde{\delta}=\left\{\begin{array}{l}
1,\left\{d E>0 \text { and } P<P_{a n}\right\} \text { or }\left\{d E<0 \text { and } P>P_{a n}\right\} \\
0, \text { otherwise } .
\end{array}\right.
$$

The parameter $k$, which quantifies the average energy required to reorient domains, is demonstrated in [13] to be asymptotically approximated by the coercive field $E_{c}$ in soft materials. 
The second component of the polarization is the reversible polarization which models the effects of domain wall bending. To first approximation, this is modeled by the relation

$$
P_{\text {rev }}=c\left(P_{a n}-P_{\text {irr }}\right)
$$

where $c$ is a parameter which must be estimated for the specific application.

The total polarization is then given by

$$
P=P_{\text {rev }}+P_{\text {irr }}
$$

To implement the model, the effective field for a given field and irreversible polarization level is computed using (1). This effective field value is then employed in either (2) or (3) to compute the corresponding anhysteretic polarization. The subsequent irreversible polarization is determined by numerically integrating (4). The total polarization is then specified by (6).

\section{Model Validation}

The polarization model (6) provides the capability for characterizing hysteresis loops which are either symmetric or asymmetric functions of the input field. To illustrate the performance of the model, we consider the characterization of both poled and unpoled PZT4 wafers. The reported results were obtained from a rectangular wafer having a length of $3.81 \mathrm{~cm}$, width of $0.635 \mathrm{~cm}$ and thickness of $0.0381 \mathrm{~cm}$. The wafer was initially poled in the transverse direction.

Input voltages ranging from $600 \mathrm{~V}$ to $2200 \mathrm{~V}$ were applied to the wafer under thermally controlled conditions and the resulting polarization levels were measured. To minimize frequency effects, the input frequencies were limited to $200 \mathrm{mHz}$ and $1 \mathrm{~Hz}$. While some frequency dependence was noted at $1 \mathrm{~Hz}$, it appears to be manifested primarily in the field attained in the material, and the model performed adequately when the field measured in the material was employed as input. We note that under quasistatic conditions, the field generated in the material by an input voltage $V$ can be approximated by the relation $E=V / d$ where $d=0.0381 \mathrm{~cm}$ is the thickness of the wafer. The validity of this relation degrades, however, as frequency effects are manifested.

Initial data sets were collected at $1 \mathrm{~Hz}$ under the biased, and hence asymmetric, conditions attributed to poling. The model parameters were estimated through a least squares fit to the $2200 \mathrm{~V}$ input data which yielded the values $\alpha=6.4 \times 10^{6} \mathrm{Vm} / \mathrm{C}, a=8.0 \times 10^{5} \mathrm{C} / \mathrm{m}^{2}, k=1.5 \times 10^{6} \mathrm{C} / \mathrm{m}^{2}$, $c=0.5, P_{s}=.44 \mathrm{C} / \mathrm{m}^{2}$ and $E_{0}=-4.0 \times 10^{5} \mathrm{~V} / \mathrm{m}, P_{0}=.01 \mathrm{C} / \mathrm{m}^{2}, P_{1}=0$. The model, with these parameter values, was then used to predict the hysteresis curves for the material using the measured field values corresponding to $1000 \mathrm{~V}, 1400 \mathrm{~V}$ and $1800 \mathrm{~V}$ inputs. The resulting model predictions are compared with the measured data in Figure 1a. It is observed that while the model over-predicts the maximum polarization for the $1000 \mathrm{~V}$ input, it characterizes the asymmetry and accurately predicts the hysteresis at the intermediate drive levels.

The wafer was then partially depoled by subjecting it to a sustained high voltage under thermally controlled conditions so that subsequent data was, at least approximately, rotationally symmetric about $E=0, P=0$ as illustrated in Figure 1b. The model with the same hysteresis parameters $a, k, c, \alpha, P_{s}$ estimated from the poled $1 \mathrm{~Hz}, 2200 \mathrm{~V}$ input data, was then used with the bias values $E_{0}=P_{0}=P_{1}=0$ to predict the hysteresis in the depoled wafer at various input levels. The data and model behavior for the $1000 \mathrm{~V}$ and $1800 \mathrm{~V}$ inputs can be compared with those in Figure 1a, and the lower voltage behavior is included to further illustrate the flexibility of the model. We note that while the model fits in Figure 1b can be improved if parameters are refined, the accuracy attained using parameters estimated under different operating conditions attests to the flexibility provided by the incorporation of associated physics in the model. 

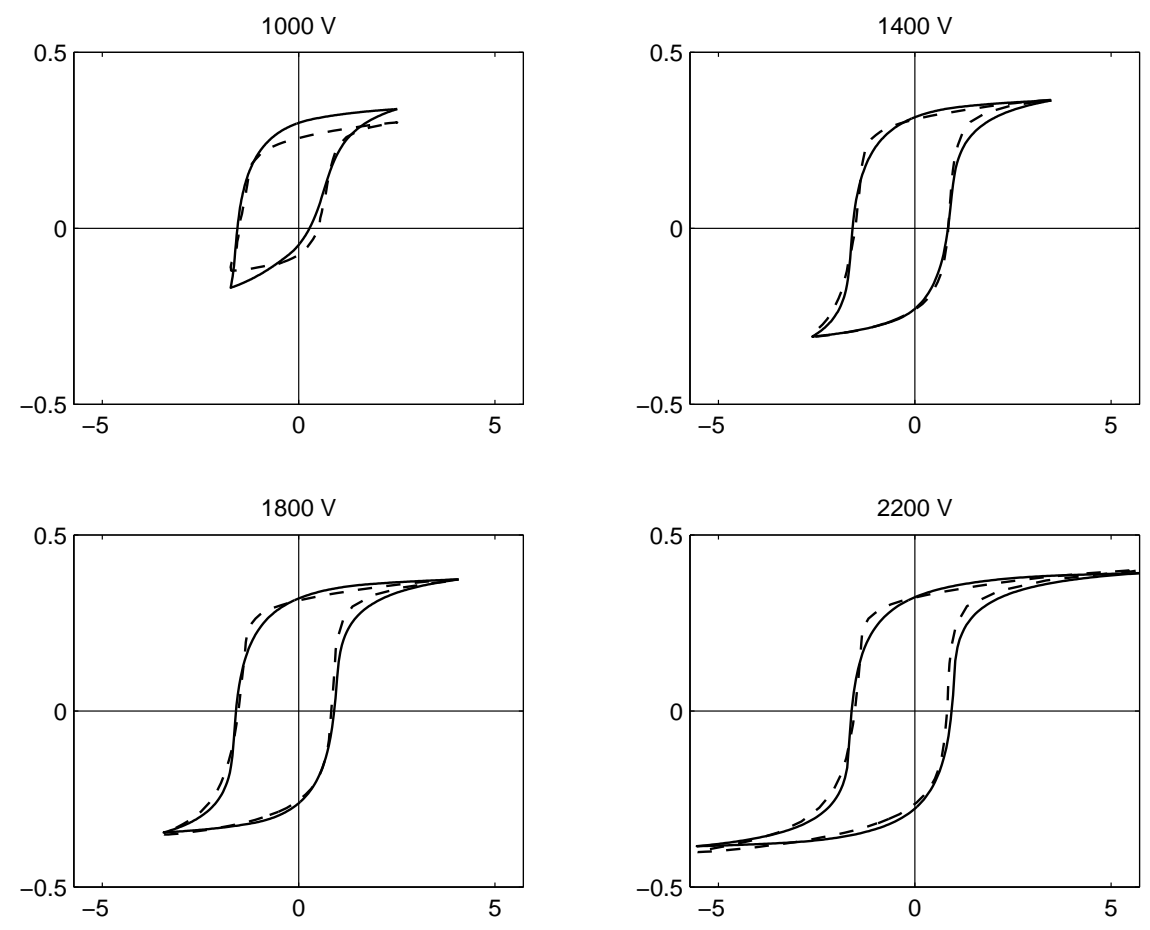

(a) Poled Wafer: Field $E$ versus Polarization $P$
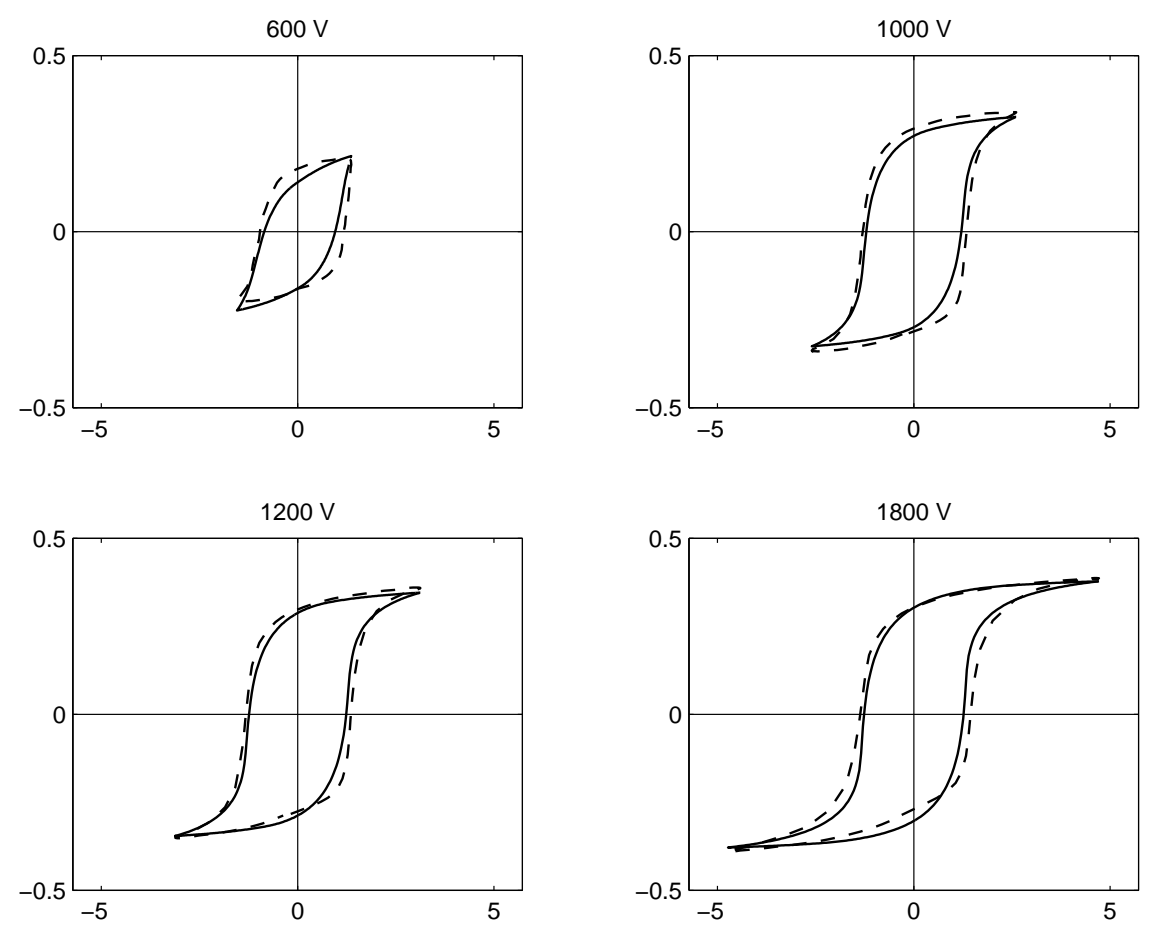

(b) Depoled Wafer: Field $E$ versus Polarization $P$

Figure 1. Model fit to asymmetric and symmetric PZT4 data with parameters estimated from poled $1 \mathrm{~Hz}, 2200 \mathrm{~V}$ input data; Model (-), Data $\left(--_{-}\right.$). (a) Fit to $1 \mathrm{~Hz}$ data for the poled wafer with $E_{0}=-4.0 \times 10^{5}, P_{0}=.01, P_{1}=0$. (b) Fit to $200 \mathrm{mHz}$ data for the depoled wafer with $E_{0}=P_{0}=P_{1}=0$. 


\section{Concluding Remarks}

This paper outlines extensions to a domain wall model for piezoelectric materials to accommodate asymmetries due to poling. This modeling strategy is based on the quantification of reversible and irreversible polarization changes due to domain wall pinning at inclusions inherent to the materials. The resulting model has five hysteresis parameters and three field and polarization parameters to incorporate biases due to poling.

The performance of the model was illustrated through a comparison with asymmetric and symmetric PZT4 data collected at several drive levels. The model parameters were estimated through a least squares fit to data collected from the poled wafer at a high drive level and the ensuing model was used to predict both asymmetric and symmetric hysteresis at several drive levels. As illustrated by the model fits, the formulation of the model based on energy principles provides it with the capability for accurately predicting the quasistatic hysteresis curves under varied poling and drive conditions.

Finally, the ODE nature of the model makes it amenable to inversion through the consideration of a complementary ODE in a manner analogous to that described in [9]. This facilitates the construction of an inverse compensator which can be used for linear control design [14].

\section{Acknowledgements}

The research of R.C.S. was supported in part by the Air Force Office of Scientific Research under the grant AFOSR-F49620-98-1-0180.

\section{References}

[1] I-W. Chen and Y. Wang, Ferroelectrics, 206, 1998, pp. 245-263.

[2] W. Chen and C.S. Lynch, J. of Intell. Mater. Syst. and Struct., 9, 1998, pp. 427-431.

[3] W.S. Galinaitis and R.C. Rogers, SPIE Conference on Mathematics and Control in Smart Structures, San Diego, CA, 1997.

[4] P. Ge and M. Jouaneh, Precision Engineering, 17, 1995, pp. 211-221.

[5] D.C. Jiles and D.L. Atherton, J. Magn. Magn. Mater., 61, 1986, pp. 48-60.

[6] B.D. Laikhtman, Soviet Physics Solid State, 15(1), 1973, pp. 62-68.

[7] J.A. Main, E. Garcia and D.V. Newton, Journal of Guidance, Control and Dynamics, 18(5), 1995, pp. 1068-1073.

[8] M. Omura, H. Adachi and Y. Ishibashi, Japanese Journal of Applied Physics, 30(9B), 1991, pp. 2384-2387.

[9] R.C. Smith, CRSC Technical Report CRSC-TR98-36; Mathematical and Computer Modeling, to appear.

[10] R.C. Smith and C.L. Hom, SPIE Conference on Mathematics and Control in Smart Structures, SPIE Volume 3667, Newport Beach, CA, March 1-4, 1999, pp. 150-161. 
[11] R.C. Smith and C.L. Hom, CRSC Technical Report CRSC-TR99-1; J. of Intell. Mater. Syst. and Struct., to appear.

[12] R.C. Smith and Z. Ounaies, ICASE Report 99-29; Proc. of the ASME Int. Mechanical Engineering Congress and Exposition, Nashville, TN, November, 1999, to appear.

[13] R.C. Smith and Z. Ounaies, CRSC Technical Report CRSC-TR99-33; J. of Intell. Mater. Syst. and Struct., submitted.

[14] G. Tao and P.V. Kokotović, Adaptive Control of Systems with Actuator and Sensor Nonlinearities, John Wiley and Sons, New York, 1996.

[15] X.D. Zhang and C.A. Rogers, J. of Intell. Mater. Syst. and Struct., 4, 1993, pp. 307-316. 